\title{
EFFECTS OF WET CUPPING THERAPY IN PATIENTS WITH MYOFASCIAL PAIN SYNDROME: A RANDOMIZED CONTROLLED TRIAL
}

\author{
${ }^{1}$ Okumus $\mathrm{M}^{1}$, Dindar $\mathrm{N}^{2}$, Kultur $\mathrm{T}^{1}$, Boyunaga $\mathrm{H}^{2}$, Rajput $\mathrm{KU}^{3}$, Keskin ED ${ }^{1}$, Dursun GA ${ }^{2}$ \\ ${ }^{1}$ Department of Physical Medicine and Rehabilitation Faculty of Medicine, University of \\ Kırıkkale-Kırıkkale, Turkey \\ 2 Department of Biochemistry, Faculty of Medicine, University of Kırıkkale- Kırıkkale, \\ Turkey \\ ${ }^{3}$ British Cupping Society-London, United Kingdom
}

\section{Introduction:}

The pathophysiology of myofascial pain syndrome (MPS) remains unclear, but energy metabolism has been implicated. A wide variety of treatment approaches are available to patients with MPS including both allopathic and complementary medicine treatments. Wet cupping therapy (WCT) has been used as an alternative treatment method throughout the world for a number of pain conditions. The aim of this study was to evaluate the effectiveness of WCT in patients with MPS and to assess the energy metabolism changes in patients with MPS before and after the WCT.

\section{Methods:}

Eighty eight patients with MPS were randomly allocated into two groups; WCT $(n=43)$ and physical therapy $(n=45)$ respectively. The WCT group received WCT twice a month whilst the control group received conventional physical therapy. Visual analogue Scale of pain (VAS-pain), Notthingham health profile (NHP) and Beck depression inventory (BDI) were used for the evaluation of pain, quality of life and depression in patients with MAS. As an indicator of energy metabolism, intra-leukocyte lactate levels in venous and cupping blood were measured before treatment and 2 weeks after treatment in the WCT group. Each WCT procedure lasted about 30 minutes and was conducted in five steps . 1.Primary sucking; The cup is placed on the selected site and the air inside the cup rarified via manual suction an is left for 3-5 minutes. 2.Scarification; Swab the treated sites with potadine solution and superficial incisions are made on the skin using sterile surgical blades for incision. 3.Bloodletting; The cup soaked with EDTA(heparin) placed back on the skin, using the same method until it was filled with blood. Each phase of WCT was divided based on a time period of 3-5 minutes. 4.Removal; The cups were removed. 5. Apply antibiotic cream and bandages, dressing

\section{Results:}

Within each group, the analysis demonstrated statistically significant improvements in VAS from baseline to 1 and 3 months $(p<0.001)$. Within each group, the analysis demonstrated statistically significant improvements in Beck depression index and Notthingam health profile (NHP) (except NHP emotional reactions sub group) and from baseline to 1 month $(p<0.05)$. There was no significant difference between the two groups in terms of efficacy of treatment ( $p>0.05)$. The lactate level in 21 of the patients in the WCT group could be measured without error. The level of lactate in the cupping blood after the WCT was significantly lower than before the WCT $(10.9 \pm 9.2 \mu \mathrm{g} / \mathrm{mL}$ versus $22.1 \pm 23.1 \mu \mathrm{g} / \mathrm{mL})(p=0.016)$. Venous blood lactate level was also low after the WCT but not statistically significant $(23.0 \pm 23.6$ $\mu \mathrm{g} / \mathrm{mL}$ versus $32.2 \pm 33.6 \mu \mathrm{g} / \mathrm{mL})(\mathrm{p}=0.122)$.

\section{Conclusion:}

We concluded that WCT is at least as effective as physical therapy in patients with MAS. As a result a tendency of anaerobic glycolysis to aerobic glycolysis intra-leukocyte cells was statistically shown by WCT. WCT may be considered beneficial by influencing energy metabolism in the treatment of patients with MPS. And future studies testing action mechanism of WCT and including control groups are needed.

Table 1. Demographic characteristics of the patients

\begin{tabular}{|c|c|c|c|}
\hline & $\begin{array}{ll}\text { WCT } & \text { group } \\
(n=43) & \end{array}$ & PT group $(n=45)$ & $p$ \\
\hline Age (mean $\pm S D$ )(year) & $47.1 \pm 8.8$ & $45.8 \pm 11.8$ & 0.563 \\
\hline Female/male & $31 / 12$ & $25 / 20$ & 0.107 \\
\hline BMI (kg/cm²) & $28.4 \pm 4.3$ & $27.5 \pm 3.2$ & 0.281 \\
\hline $\begin{array}{l}\text { Symptom } \\
\text { duration(mean } \pm S D)(\text { month) }\end{array}$ & $5.9 \pm 4.1$ & $6.6 \pm 4.3$ & 0.447 \\
\hline
\end{tabular}

Table 2. Intra-leukocyte lactate levels in venous and cupping blood before treatment 2 weeks after treatment $(n=21)$

\begin{tabular}{llll}
\hline & \multicolumn{3}{c}{ Second } \\
& Baseline & week & $\mathrm{p}$ \\
\hline Lactate levels-CB $^{*}$ & $22.1 \pm 23.1$ & $10.9 \pm 9.2$ & 0.016 \\
Lactate levels-VB* & $32.2 \pm 33.6$ & $23.0 \pm 23.6$ & 0.122 \\
\hline CB: cupping blood, VB: Venous blood, $\mathrm{p}>0.05,{ }^{*} \boldsymbol{\mu g} / \mathrm{mL}$
\end{tabular}

